\title{
Inverse Problems in Optics
}

\author{
H.P. Baltes, Zug \\ (Zentrallabor, Landis \& Gyr)
}

tered radiation field, such as the angular distribution of radiated energy or the resulting photon counting statistics. The inverse problem is to deduce features of radiation sources or scatterers from detectable properties of the emitted or scattered field. Given, for example, some far-field data (usually up to some error and noise), the aim is to infer properties of the source or the scatterer.

Fig. 1 - PHONOCARD station and cards lby courtesy of SODECO-SAIAJ.

Can one reconstruct the shape of a scattering object from data on the scattered radiation field? Is it possible to design a grating with a groove profile, tailored to diffract determined fractions of the incident radiant energy into defined diffractionorder directions? Do optical correlation measurements permit detection of the structure of a scattering object that is hidden behind a diffuse layer? These are typical questions within the field of optical physics that come under the heading of inverse source and scattering problems - an area of strong current scientific interest ${ }^{1,2}$ ).

The present concern is, however, not motivated only by scientific curiosity, but also by industrial demands. For instance, specific development problems pursued in the author's laboratory include the encoding of information in tailored diffracting or scattering structures, the reading of such information using detectors, and the retrieval of structural information from given random scatterers (such as a rough surface) with emphasis on the automatic optical checking of identify or authenticity $^{3}$ ). One example of a product based on such development is the PHONOCARD ${ }^{\circ}$ currently being manufactured by SodecoSaia, a company of the Landis \& Gyr group $\left.{ }^{4}\right)$. The PHONOCARD ${ }^{\circ}$ is a pay telephone operated by pre-paid cards with optically stored value units, whose authenticity is checked by an optical reading system, Fig. 1.

What distinguishes the "inverse" or "indirect" problem in optical physics from the "direct" or "normal"? The direct problem is to predict the emission or propagation of radiation on the basis of a known arrangement of sources or scatterers. Given the source distribution or the incident radiation field and the structure of the scatterer, one seeks to calculate properties of the scat-

\section{Mathematical Estimates}

An intuitive solution of optical inverse problems is common place: we infer the size, shape, surface texture and material of objects from the manner in which they scatter and absorb light as detected by the eyes. Intuition, however, has to give way to mathematical reconstruction or estimates as soon as we wish to analyse optical data beyond their visual appearance. Examples are the extrapolation (beyond the boundary) and deblurring of optical images, the retrieval of structural information from intuitively incomprehensible data such as defocussed images or interferograms, or the search for information that is "lost" in the detection process such as the phase.

A distinctive feature of inverse problems is that they are usually more difficult to solve than the corresponding direct problems, which are somewhat closer to textbook physics. Mathematically speaking, the solution of an inverse problem requires the inversion of the relationship (e.g. described in terms of an integral equation) between the structure of the scatterer and the observed data on the scattered field. The problem of inverting such a relationship inevitably leads to questions on the uniqueness and stability of the inversion (if these exist at all). There can be many different objects, e.g. grating groove profiles, that produce the same scattering data (at least within the accuracy of measurement) and small errors in the data can lead to large errors in the reconstruction of the scatterer. Sometimes (but not always) these problems of non-uniqueness and instability can be solved by exploiting additional information such as prior knowledge or further data from other experiments.

Apart from the mathematical interest, the question of uniqueness is important for many applications of inverse scattering, e.g. the optical checking of scattering structures against possible counterfeits.

\section{Deterministic and Statistical Problems}

There are many different inverse problems in optical physics, each with its own subtleties. It is perhaps useful to distinguish between the "scientist's inverse problems" - the retrieval from measured data of features characterising particular scatterers or sources - and the "engineer's inverse problems" - to design an object with a structure that yields certain wanted scattering properties.

Another useful classification can be based on the statistical character of the radiation field and the information to be retrieved. Deterministic problems involve coherent radiation with a well-defined (complex) amplitude, and scatterers with a well-defined structure; statistical problems deal with partially coherent radiation fields and stochastic scatterers such as rough surfaces or fluctuating media. Partially coherent radiation cannot be described in terms of a well-defined complex amplitude, but rather in terms of averages, moments, and correlations.

The retrieval of the phase of a coherent wave from the known intensity distribution, the determination of a scattering potential of a dielectric object, or the question of estimating details of sources or scatterers beyond the classical diffraction limit, are examples of "deterministic" inverse problems. Typical "statistical" inverse problems are the reconstruction of the size of a source (e.g. a remote star) from the far-zone degree of coherence as measured by an interferometer, the retrieval of averages and correlations characterising a disordered scattering system from the statistical properties of the scattered radiation, or the estimate of the photon statistics of the radiation field received by a detector from raw photoelectric detection data. The state of the art of a number of different inverse problems in optical physics has recently been reviewed in two books ${ }^{1,2}$ ) edited by the author.

Two specific inverse problems that have been studied in the author's laboratory in collaboration with the Institut de Physique Théorique, of the Ecole Polytechnique Fédérale of Lausanne, the Department of Applied Physics, the State University 


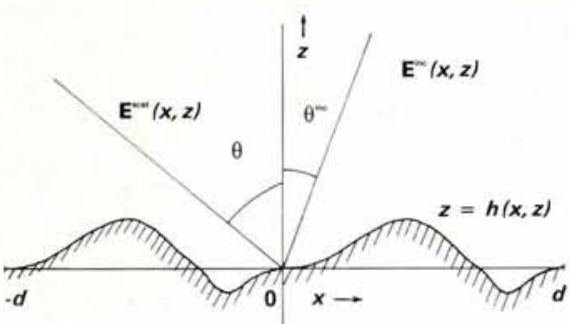

Fig. 2 - The scattering geometry for the grating profile reconstruction problem. IAfter Optics Communs. 41 (1982) 149).

Groningen, and the Institute of Physics and Atmospheric Sciences, Drexel University, Philadelphia, will be cited. One is a deterministic problem on the level of electromagnetic inverse scattering, namely the design of grating profiles tailored to yield certain desired diffraction efficiencies (i.e. the relative radiant intensity that is scattered into the various diffraction orders). The other is a problem of statistical optics, namely the question whether a grating hidden behind a diffusor can be reconstructed by observing the degree of coherence of the scattered light.

\section{Construction of Grating Profile}

The scattering geometry is shown in Fig. 2 where the incident field is denoted by $E^{\text {inc }}(x, z)$ and the scattered field by $\mathbf{E}^{\text {scat }}(x, z)$; the unknown grating profile is described by the function $z=h(x)$. Invariance is assumed in the $y$ direction. Under the assumption of perfectly conducting grating material, the profile $z=h(x)$ has to be determined such that the boundary condition

$\left[\mathrm{E}^{\text {inc }}(x, z)+\mathrm{E}^{\text {scat }}(x, z)\right]_{z=h(x)}=0$ is fulfilled. Assuming an incident plane wave of monochromatic radiation (wavelength $\lambda$ ) polarized parallel to the $y$ direction, the scattered field can be shown to have the same polarisation. Far away from the grating, the scattered field can be represented as a superposition of a number $N$ of plane waves travelling in different directions according to Bragg's law, with coefficients $c_{n^{\prime}} n=1, \ldots, N$. The relative amount of energy propagated into each direction is given by the corresponding "efficiency" $\left|c_{n}\right|^{2}$. The problem is to determine a profile $h(x)$ from given values of $\left|c_{n}\right|^{2}$.

\section{Reconstruction Procedure}

In a two-step reconstruction procedure recently devised by A. Huiser, A. Quattropani, and the author, the scattered field was assumed to be approximately represented by a superposition of plane waves also close to the grating, (technically known as the Rayleigh hypothesis). Hence equ. (1) should hold approximately for that representation of $\mathbf{E}^{\text {scat }}(x, z)$ also, and one may expect to find a first approximation $h_{1}(x)$ of $h(x)$ from minimizing the functional

$F \equiv \int d^{2} s\left|E^{\text {inc }}\left(x, h_{1}(x)\right)+\mathbf{E}^{\text {scatt }}\left(x, h_{1}(x)\right)\right|^{2}$

with $d^{2} s=d^{2} x+d^{2} h_{1}$

The second step consists in adding a finite number $M$ (say $M=20$ ) of so-called evanescent waves (i.e. waves with exponential damping in the $z$ direction) to the previous expression for the scattered field. Their wavelength is $\lambda$ and their decrement is of the order of a few $\lambda^{-1}$. The relative importance of these waves is controlled by yet unknown coefficients $a^{1}{ }_{m}, m=1, \ldots$, $M$. We are quite entitled to add these waves as their contribution to the scattered field is negligible in the far zone. Using the previous first approximation $h_{1}(x)$ for the grating profile, we determine the coefficients $a^{1}$ by minimizing the functional (2) within the new representation of $\mathbf{E}^{\text {scat }}(x, z)$. With $a_{m}^{1}$ thus determined, we find an improved profile $h_{2}(x)$ by again minimizing the functional with respect to $h(x)$ and using the improved profile $h_{2}(x)$, we determine improved coefficients $a^{2}{ }_{m^{\prime}}$ and so forth.

\section{A Non-unique Reconstruction}

The consistency of the procedure can be checked by starting from the efficiencies $\left|c_{n}\right|^{2}$ corresponding to some known profile and checking whether this profile is indeed reconstructed. For this purpose consider the profile shown in the middle of the right hand side of Fig. 3. The first step of the procedure is illustrated on the left hand side of the same Fig., where the following function is plotted:

$$
f(x, z)=\left|E^{\text {inc }}(x, z)+\mathbf{E}^{\text {scat }}(x, z)\right|^{2}
$$

Dark areas correspond to large and light areas to small values of the function (3). Any curve connecting only light areas from left to right can be considered as a first approximation $h_{1}(x)$ of the profile, because along such a curve the boundary condition (1) is approximately fulfilled. Each of these curves provides a local minimum of the functional (2).
Apparently, the procedure is not unique. Two different types of first-approximation profiles $h_{1}(x)$ occur. After 10 iterations of the above two-step procedure, they lead to the reconstructed profiles $A$ and $B$ shown in Fig. 3. While profile $B$ is very close to the original profile, curve $A$ represents a quite different profile. Within the precision of the computation, both profiles $\mathrm{A}$ and $\mathrm{B}$ produce the same efficiencies.

\section{Relevance of Far-zone Coherence}

The structure of a source or scatterer manifests itself not only in the far-zone distribution of the energy of the scattered radiation, but also in the far-zone degree of coherence $\mu$, whose absolute value $|\mu|$ is the fringe contrast that the scattered radiation produces if subject to appropriate interferometric experiments. We recall that completely incoherent radiation leads to zero fringe contrast, fully coherent radiation to maximum contrast, and partially coherent radiation to something in between.

It is well-known that observation of the far-zone coherence can sometimes provide a tool more powerful than measurement of the angular energy distribution for the detection of characteristic features of sources or scatterers. A famous example is the determination of the size of stars from measurements of $|\mu|$ as a function of angular spacing using the Van Cittert-Zernike (VCZ) theorem. In the case of an incoherent source, the far-zone degree of coherence is essentially given by the Fourier transform of the source brightness profile, i.e. the intensity as a function of position in the source plane ${ }^{5}$ ). Assuming as "prior knowledge" that the star is a uniformly bright, circular disk of poor coherence, its (angular) diameter can be determined from the first zero of $|\mu|$, even when the size

Fig. 3 - Non-uniqueness of profile reconstruction. See text for explanation. IAfter Optics Communs. 41 (1982)].
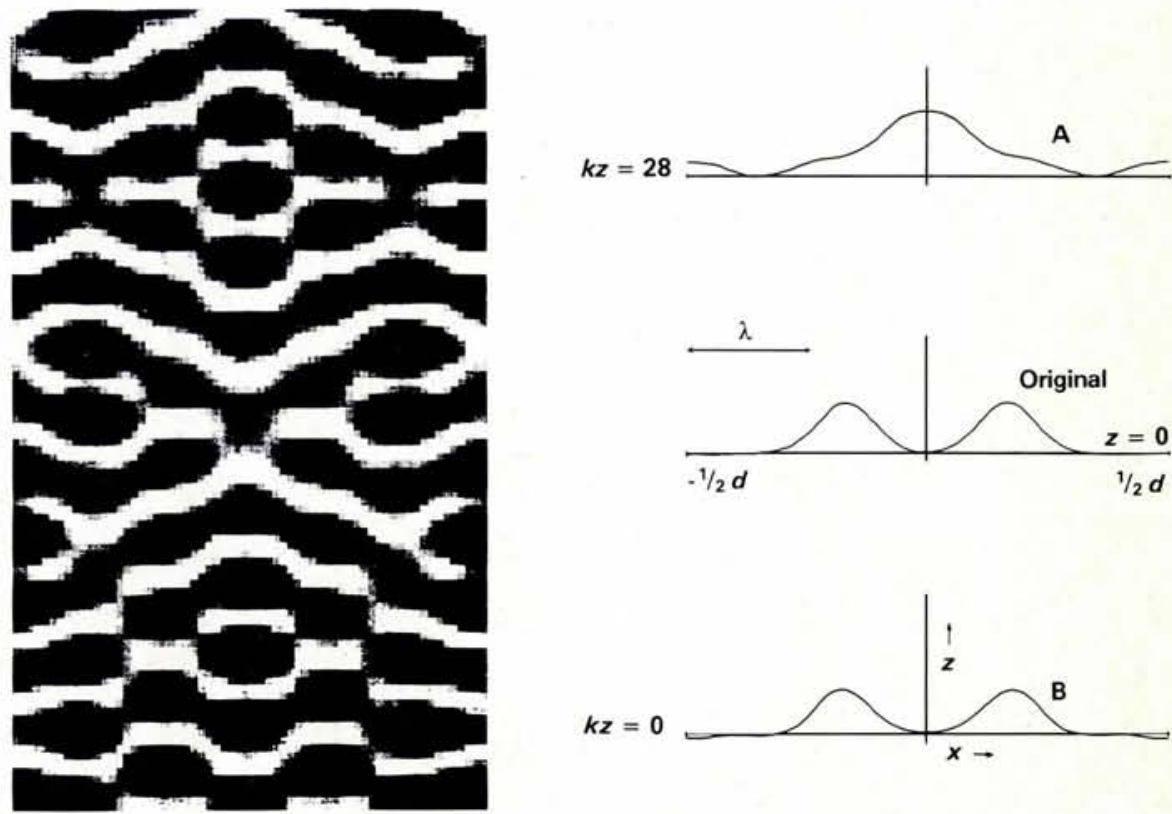

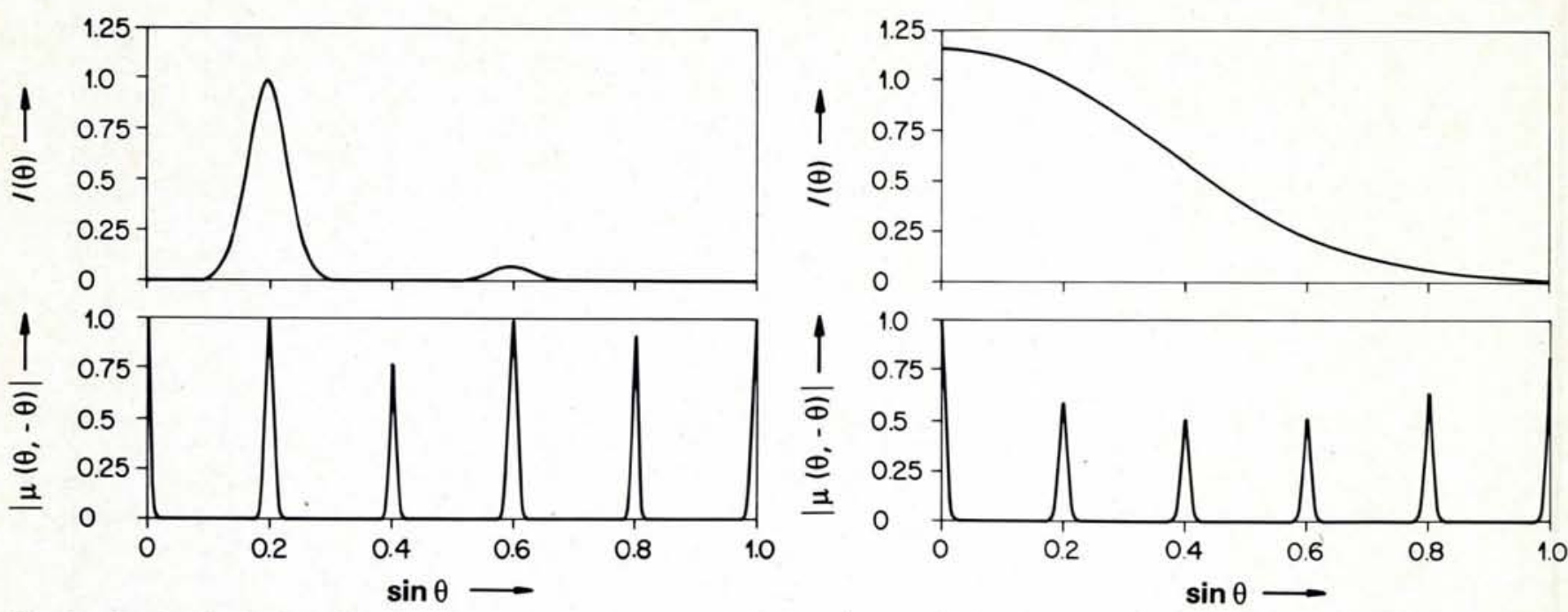

Fig. 4 - Angular distribution of far-zone intensity $((\theta)$ and degree of coherence $|\mu(\theta,-\theta)|$ of radiation emanating from a lamellar phase grating hidden behind a diffuser. See text for explanation. [After Optica Acta 29 (1981) 169].

cannot be resolved by direct telescope observation.

\section{Phase Gratings Hidden Behind a Diffu- sor}

The situation of a phase grating embedded in a diffuse fluctuating medium or hidden behind a rotating ground glass is somewhat similar to that considered above, the illuminated diffuser acting as the incoherent source of the VCZ theorem. There are, however, two important differences. First, the object to be detected is a phase object. Thus the classical VCZ theorem would yield only a trivial far-zone degree of coherence, furnishing information about the width of the illuminating laser beam, but not the presence of the grating. Second, the diffuser may be characterized by a small, but non-zero correlation length / leading to partially coherent, and not fully incoherent radiation, as required for the $\mathrm{VCZ}$ theorem.

The theoretical investigation of the farzone degree of coherence of a phase grating behind a diffuser illuminated by a laser beam was initiated in 1979 by H.A. Ferwerda, B. Steinle, and the author and was further pursued by A.S. Glass. The results suggest that interferometry may in fact permit the detection of source periodicity (e.g. the presence of a phase grating) which might be undiscernible otherwise because the radiated intensity is too diffuse.

Typical results are shown in Fig. 4 where the angular distribution $/(\theta)$ of the scattered intensity in comparison with the absolute value, $|\mu(\theta,-\theta)|$, of the far-zone degree of coherence, in a plane perpendicular to the grating grooves. The period of the underlying phase grating (a lamellar reflection grating) is $b=5 \lambda, \lambda$ denoting the wavelength. In the left hand diagrams, the diffuser is characterized by a moderately short correlation length /. Because of the diffuser, broad diffraction lobes $/(\theta)$ are obtained instead of sharp diffraction peaks, but different orders can still be resolved. In the right hand diagrams, a stronger diffuser with much shorter correlation length wipes out any structure in the $/(\theta)$ curve. However, the "hidden" periodicity still manifests itself in the sidepeaks of the $|\mu(\theta,-\theta)|$ curve. The width of these "coherence peaks" is predicted to be inversely proportional to the (large) width of the illuminating laser beam.

Recent interferometric experiments carried through by K.M. Jauch during a stay at Drexel University aimed at the demonstration of the first side maximum in the $|\mu(\theta,-\theta)|$ diagram, i.e. at the detection of a fringe contrast maximum between beams with an angular separation corresponding

Fig. 5 - Experimental set-up for the detection of side-maximum in the far-zone degree of coherence. See text for explanation. (After Optica Acta 28 (1981) 1013).

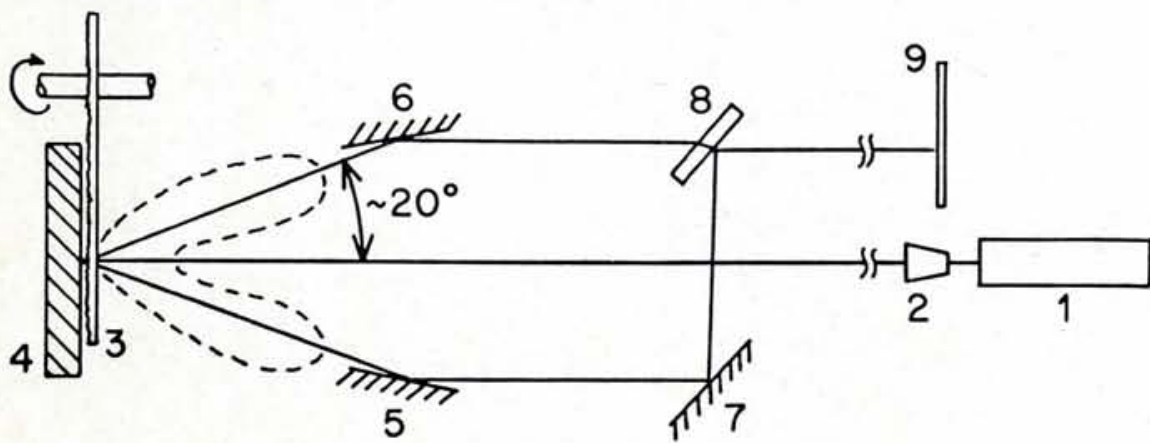

to that between the first and minus first diffraction orders of the underlying grating. The results are in accordance with the theoretical predictions. A typical experimental setup is shown in Fig. 5. It consists of a laser 1 , a beam expander 2 , a rough surface 3 , a grating 4 , mirrors $5,6,7$, beam splitter 8 and an observation screen 9 . In particular, the width of the $|\mu|$ peak was found to be inversely proportional to the beam width as predicted.

An alternative way of detecting the hidden grating would be to measure the degree of far-zone intensity correlation by a photo-counting experiment.

\section{REFERENCES}

1. Baltes, H.P., Ed., Inverse Source Problems in Optics (Springer-Verlag), 1978.

2. Baltes, H.P., Ed., Inverse Scattering Problems in Optics (Springer-Verlag), 1980.

3. Greenaway D.L., Landis \& Gyr Review, 27 (1980) No. 1, p. 20

4. Wiblé P., Landis \& Gyr Review, 27 (1980) No. 1, p. 39.

5. See e.g.: Klauder, J.R., Sudarshan E.C.G., Fundamentals of Quantum Optics (Benjamin 1968), or Chap. 5 in Ref. 1.

\section{Hewlett-Packard Europhysics Prize}

Members, acting individually or as the representative of a Division are invited to submit nominations for the 1983 HewlettPackard Europhysics Prize and include:

a clear definition of the work in question; a short biography of the candidate; a list of relevant publications and reprints referring to the work recommended.

Details should be sent to the Selection Committee, c/o EPS Secretariat, POB 69. $\mathrm{CH}-1213$ Petit-Lancy 2, to arrive not later than 15 August 1982. All information will be treated as confidential. The Committee cannot enter into correspondence over the selection made. 